\title{
La relación entre el presupuesto participativo y la ejecución presupuestaria de la Municipalidad Distrital de Lurigancho del año 2016
}

The relationship between the participatory budget and the budget execution of the District Municipality of Lurigancho for the year 2016

\begin{abstract}
RESUMEN
El presente artículo, titulado "La relación entre el Presupuesto Participativo y la Ejecución Presupuestaria de la Municipalidad Distrital de Lurigancho del año 2016", tiene como problema general que impulsa la investigación del artículo, la aplicación de la siguiente pregunta: ¿Cuál es la relación entre el Presupuesto Participativo con la Ejecución Presupuestaria de la Municipalidad Distrital de Lurigancho, durante el ejercicio 2016?; de igual forma, el objetivo general es precisar en qué medida el Presupuesto Participativo (PP) está vinculado a la Ejecución Presupuestaria (EP) en el distrito de Lurigancho. Los resultados fueron la alta correlación existente entre el presupuesto participativo con la ejecución del presupuesto del Municipio de Lurigancho, utilizando el RHO de Spearman. Así pues, este artículo se encuadra dentro del diseño transversal, correlativo, descriptivo, no experimental y cuantitativo. Es importante señalar que se empleó una población de 36 personas, conformada por 28 agentes involucrados en el proceso de presupuesto participativo y ocho empleados del Municipio de Lurigancho, donde se emplearon las herramientas adecuadas priorizando el cuestionario.
\end{abstract}

Palabras Clave: Ejecución Presupuestaria; Presupuesto Institucional de Apertura (PIA); Presupuesto Participativo.

\begin{abstract}
This article, entitled "The relationship between the Participatory Budget and the Budgetary Execution of the District Municipality of Lurigancho in 2016", has as a general problem that drives the research of the article, the application of the following question: What is the relationship between the Participatory Budget with the Budgetary Execution of the District Municipality of Lurigancho, during the fiscal year 2016; likewise, the general objective is to determine to what extent the participatory budget is linked to the Budgetary Execution in the district of Lurigancho. The results were the high correlation existing between the participatory budget with the budget execution of the Municipality of Lurigancho, using Spearman's
\end{abstract}

\section{William Adolfo Pacchua Guerra} williampg64@gmail.com Municipalidad Distrital de Surquillo. Lima, Perú

Presentado: 04/03/2020 - Aceptado: 30/04/2021 - Publicado: 23/12/2021

(C) Los autores. Este artículo es publicado por la revista Gestión en el Tercer Milenio de la Facultad de Ciencias Administrativas Universidad Nacional Mayor de San Marcos. Este es un artículo de acceso abierto, distribuido bajo los términos de la licencia Creative Commons Atribución 4.0 Internacional (CC BY 4.0) [https://creativecommons.org/licenses/by/4.0/deed.es] que permite el uso, distribución y reproducción en cualquier medio, siempre que la obra original sea debidamente citada de su fuente original. 
RHO. Thus, this article is framed within the cross-sectional, correlational, descriptive, non-experimental and quantitative design. It is important to point out that a population of 36 people was used, made up of 28 agents involved in the participatory budget process and eight employees of the Municipality of Lurigancho, where the appropriate tools were used, prioritizing the questionnaire.

Keywords: Budget execution; Opening Institutional Budget (PIA); Participatory Budget.

\section{INTRODUCCIÓN}

Una adecuada Ejecución Presupuestaria del Presupuesto Participativo requiere de un gran compromiso e involucramiento entre el gobierno local y la sociedad civil, fomentando la importancia del papel del ciudadano como líder de opinión y decisión, además de responsable de las decisiones tomadas de manera colectiva para acceder a las necesidades básicas insatisfechas y mejoramiento de situaciones de vida (Páez-Álvarez, 2006). Es por ello que, el presente articulo pretende identificar la relación existente entre la Ejecución Presupuestaria y el Presupuesto Participativo en la Municipalidad Distrital de Lurigancho.

Desde un inicio, el proceso de Presupuesto Participativo (Orçamento Participativo, en portugués) en Brasil, ha cambiado en las administraciones locales la democracia participativa en un modelo internacional (Estambul, Hábitat II, 1996), asimismo "ha sugerido el empleo de los procedimientos de Presupuestos Participativos en las jefaturas locales y avalaba a este modelo de procesos como uno de los ejercicios más relevantes de reemplazo citadino a nivel global" (Blanco, 2002, p. 1).

Estudios como el de Musgrave, Keynes y Smith, señalan que: "aspectos como la ausencia de transparencia, la buena planeación y el deficiente conocimiento técnico y estratégico por parte de la burocracia, forman un escenario donde el vínculo entre inversión pública y crecimiento puede no resultar automático" (Tabuca, 2016, p. 128). Así como Rojas (2015) quien en su estudio resaltó que la falta de comunicación se puede ver influenciada por factores externos como interferencias políticas en delegados, dirigentes y empleados públicos.

El economista brasileño Ubiratan de Souza, define al presupuesto participativo, así:
Es un proceso de democracia directa, voluntaria y universal, donde el ciudadano puede discutir y decidir sobre el presupuesto y las políticas públicas. El ciudadano no limita su participación, sino que también decide las prioridades de gastos y controla la gestión del gobierno. Deja de ser un coadyuvante de la política tradicional para ser el personaje principal y permanente de la gestión pública. El Presupuesto Participativo combina la democracia directa con la democracia representativa, una conquista a ser preservada y calificada. (UN-HABITAT, 2004, p. 20)

Siendo un artículo en la línea de Gestión Pública, se tiene que mencionar lo que el marco legal establece con relación al Presupuesto Participativo, siendo la "Ley Marco del Presupuesto Participativo", Ley $\mathrm{N}^{\circ} 28056$, que establece:

[Como] un mecanismo de asignación racional, eficiente, equitativa, eficaz y transparente de los recursos públicos, que fortalece las relaciones Estado - sociedad civil; para lo cual los gobiernos regionales y locales promueven el desarrollo de estrategias y mecanismos de participación en la programación de sus presupuestos, así como en la fiscalización y vigilancia de la gestión de los recursos públicos". (Ley $\mathrm{N}^{\circ} 28056$, 2003, p. 249441)

Además, existen indicadores que mejoran la visión del Presupuesto Participativo, estos son: la participación, que se enfoca en la intervención de trabajos para la mejoría de la calidad de vida, bajo un modo proactivo y funcional cuyos beneficios se dan en conjunto (Sánchez, 2000; Linale 2006); la transparencia, cuya información las instituciones por ley 
están obligadas a ponerlas a disposición del público en general a través de acceso a la información (Agurcia, 2011); y la igualdad, que es un valor necesario en los sistema políticos para cimentar el Estado y democracia, que comprende más allá de las diferencias y a tener iguales oportunidades de acceso al bienestar (Montoya, 2007; Fun. Luis Vives, 2010)

En Perú, el Presupuesto Participativo, mantiene una desunión con la realidad, siendo en su fase de concertación y coordinación, que parce alejarse de los objetivos estratégicos plasmados dentro de los planes de desarrollo concertados (PDC) de los tres niveles de gobierno (nacionales, regionales y locales), del mismo modo de los Planes Estratégicos Institucionales (PEI). Por lo antes mencionado se llega a establecer que no se encuentra articulado o ajustado con los proyectos y las políticas públicas de los tres niveles de gobierno, por lo que persisten las deficiencias más sentidas, y esto se debe a la ausencia de logros visibles y socialmente aceptados, por los ciudadanos.

Tal como Gambini (2011), quien realizó un estudio en Tacna, evidenciando una relación importante sobre la participación en el proceso del proceso del Presupuesto Participativo, así como el nivel de satisfacción de los ciudadanos, ya que el escaso conocimiento sobre los beneficios y los planes inadecuados de información acerca de la gestión, generaron dificultad para incluirse al Presupuesto Participativo y la desaprobación de los habitantes a los proyectos en sus distritos.

En consecuencia, el Municipio de Lurigancho, adoptó el desarrollo del Presupuesto Participativo, como política participativa del desarrollo local y una buena gestión municipal, de igual forma reforzando los espacios de participación y concertación, con la intensión de que sus ciudadanos desatendidos sea prioritario la ejecución de las inversiones de acuerdo a sus necesidades por parte de sus representantes, esto con el fin de mejorar la ejecución presupuestaria en relación a los proyectos de inversión pública seleccionados y alcanzar que estén igualmente vinculados a las necesidades de dichos pueblos y orientados a reducir las brechas en todo el distrito de Lurigancho.
A pesar de ello, en el ejercicio fiscal 2016 se apreció que el Municipio de Lurigancho no había cumplido con esta intención, ya que encontró dificultades para ejecutar lo establecido en el Presupuesto Participativo (PP), las cuales se describen a continuación:

a. Los que participaron en el desarrollo del PP, llámese agentes participantes, ciudadanos, así como el equipo técnico, no priorizan de forma adecuada y articulada los proyectos de inversión pública (PIP) los cuales no tienen conexión con los problemas más comunes y cotidianos que aquejan a la población, que fueron identificados y priorizados en los ejes estratégicos establecidos en el Plan de Desarrollo Concertado Local (PDCL).

b. En relación a los montos determinados para los PIP aceptados y priorizados durante el desarrollo del PP, cotejado con el Presupuesto Institucional de Apertura (PIA), se observa la existencia de excesivas modificaciones presupuestarias.

c. El conjunto de aquellos proyectos priorizados durante el Presupuesto Participativo fueron propuestos y aprobados a nivel de ideas, dicho de otra manera, que no se encuentran registrados en el banco de proyectos de la municipalidad, es decir, no se hallaron declarados viables, el cual es necesario para la ejecución del mismo.

d. Existe una propensión a dar prioridad a los proyectos de infraestructura, dejando de dar prioridad a los proyectos de desarrollo de capacidades y de carácter social y la prevención del riesgo de desastres, siendo el más relevante.

Por estas razones, aquellos PIP que fueron priorizados en el desarrollo del $P P$, y agregados en el PIA, no presentan ejecución de gasto en el ejercicio 2016.

Por otro lado, el Argentino Marcos P. Makón, Contador Público de profesión, establece que la Ejecución Presupuestaria: 
Implica la movilización de todo el aparato administrativo público, utilizando las más diversas técnicas y disciplinas. Intervienen además activamente los sistemas de recursos humanos y compras y de contrataciones, así como la contabilidad que registra las transacciones económico-financieras originadas en el presupuesto. (Makón, 1999, p. 19)

De igual forma según el Capítulo II, artículo 33, Ejecución Presupuestaria, , mencionado en el Decreto Legislativo $N^{\circ} 1440$ (2018), determina:

[Que] la Ejecución Presupuestaria, se inicia el 1 de enero y finaliza el 31 de diciembre de cada año fiscal, periodo en el que se perciben los ingresos públicos y se atienden las obligaciones de gasto de conformidad con los créditos presupuestarios aprobados en las Leyes Anuales de Presupuesto del Sector Público y sus modificaciones. (Decreto Legislativo. No 1440, 2018, p. 62)

\section{MÉTODOS}

La presente investigación fue de tipo No Experimental, Cuantitativo, Descriptiva, Transversal y Correlacional. La unidad de Análisis fueron los Agentes Participantes del Procesos del Presupuesto Participativo a quienes se les administró una encuesta estructurada que incluyó 36 preguntas siendo estas de tipo cerradas.

La muestra considerada de la investigación abarcó a 36 personas, 28 fueron agentes participantes, participantes del Presupuesto
Participativo organizado por el Municipio de Lurigancho y 8 fueron empleados integrantes del equipo técnico, cuya selección se realizó a través de un muestreo aleatorio simple utilizando como marco muestral la lista de los agentes participantes inscritos en proceso del PP.

Dentro de los principales indicadores de las variables presentes en el cuestionario se incluyeron la Participación, Transparencia e Igualdad, relacionados al Presupuesto Participativo y los Ingresos Públicos, Gastos Públicos y Modificaciones Presupuestales relacionados a la Ejecución Presupuestaria. El trabajo de recogida de datos fue realizado por el autor, en el cual los encuestados solo respondieron a las preguntas relacionadas con la tipología y forma del cuestionario.

El análisis de datos fue realizado por el Software Estadístico SPSS V.25.00, utilizando tablas de frecuencias de distribución para el análisis de cada pregunta relacionada a los indicadores de cada variable de la presente investigación.

\section{RESULTADOS}

Fueron encuestados 36 personas, donde los resultados se muestran a continuación el cual se exponen las características específicas y generales, respecto a aspectos vinculados a los objetivos de la investigación, relacionado a la población elegida.

Se evidencia en la Tabla 1, el $42 \%$ contestaron que el presupuesto participativo casi nunca es utilizado, asimismo, el $44 \%$ refiere

Tabla 1

Distribución de frecuencias y porcentajes de las variables Presupuesto Participativo con la Ejecución Presupuestaria de la Municipalidad Distrital de Lurigancho en el año fiscal 2016

\begin{tabular}{|c|c|c|c|c|c|c|}
\hline & & & \multicolumn{3}{|c|}{ Y: Ejecución Presupuestaria } & \multirow{2}{*}{ Total } \\
\hline & & & Casi nunca & Ocasionalmente & Casi siempre & \\
\hline \multirow{8}{*}{$\begin{array}{l}\text { X: Presupuesto } \\
\text { Participativo }\end{array}$} & \multirow{2}{*}{ Casi nunca } & $\mathrm{F}$ & 15 & 0 & 0 & 15 \\
\hline & & $\%$ & $42 \%$ & $0 \%$ & $0 \%$ & $42 \%$ \\
\hline & \multirow{2}{*}{ Ocasionalmente } & $\mathrm{F}$ & 1 & 13 & 2 & 16 \\
\hline & & $\%$ & $3 \%$ & $36 \%$ & $6 \%$ & $44 \%$ \\
\hline & \multirow{2}{*}{ Casi siempre } & $\mathrm{F}$ & 0 & 1 & 4 & 5 \\
\hline & & $\%$ & $0 \%$ & $3 \%$ & $11 \%$ & $14 \%$ \\
\hline & \multirow{2}{*}{ Total } & $\mathrm{F}$ & 16 & 14 & 6 & 36 \\
\hline & & $\%$ & $44 \%$ & $39 \%$ & $17 \%$ & $100 \%$ \\
\hline
\end{tabular}

Nota F= Frecuencia, resultados del procesamiento mediante el programa SPSS. Elaboración propia. 
que ocasionalmente se emplea el presupuesto participativo (PP), el 14\% consideró que casi siempre se emplea el presupuesto participativo, de igual forma el $42 \%$, contestó que casi nunca realizan la ejecución del presupuesto; seguido de un $36 \%$ que respondió ocasionalmente la ejecución presupuestaria es aplicada. Se finaliza mencionando que, un $11 \%$ afirma que la ejecución presupuestaria (EP) casi siempre se aplica.

Se evidencia en la Tabla 2, que del $33 \%$ que respoSe evidencia en la Tabla 2 , que el $33 \%$ contestó que la participación casi nunca es aplicada, seguido de un $44 \%$ contestaron que la participación ocasionalmente se aplica, seguido del 17\% el cual aseguraron que la participación casi siempre se aplica, ahora bien, asimismo un $6 \%$ respondió que la participación siempre se emplea, de igual forma un 31\% afirma que casi nunca utilizan la ejecución del presupuesto, por otra parte, el $31 \%$ respondió que la ejecución presupuestaria ocasionalmente es empleada, asimismo, solo un $11 \%$ refiere que la ejecución presupuestaria casi siempre se aplica, Finalmente, un 6\% respondió que la ejecución presupuestario siempre es utilizada.

Ahora bien, en la Tabla 3, el 50\% contestaron que la transparencia casi nunca es utilizada, asimismo, el $42 \%$ refiere que ocasionalmente se emplea la transparencia, el 8\% consideró que casi siempre se emplea la transparencia, de igual forma el $31 \%$, contesto que casi nunca se

Tabla 2

Distribución de frecuencias y porcentajes del indicador Participación con la variable Ejecución Presupuestaria de la Municipalidad Distrital de Lurigancho en el año fiscal 2016

\begin{tabular}{|c|c|c|c|c|c|c|}
\hline & & & \multicolumn{3}{|c|}{ Y: Ejecución Presupuestaria } & \multirow{2}{*}{ Total } \\
\hline & & & Casi nunca & Ocasionalmente & Casi siempre & \\
\hline \multirow{10}{*}{ X1: Participación } & \multirow{2}{*}{ Casi nunca } & $\mathrm{F}$ & 11 & 1 & 0 & 12 \\
\hline & & $\%$ & $31 \%$ & $3 \%$ & $0 \%$ & $33 \%$ \\
\hline & \multirow{2}{*}{ Ocasionalmente } & $\mathrm{F}$ & 5 & 11 & 0 & 16 \\
\hline & & $\%$ & $14 \%$ & $31 \%$ & $0 \%$ & $44 \%$ \\
\hline & \multirow{2}{*}{ Casi siempre } & $\mathrm{F}$ & 0 & 2 & 4 & 6 \\
\hline & & $\%$ & $0 \%$ & $6 \%$ & $11 \%$ & $17 \%$ \\
\hline & \multirow{2}{*}{ Siempre } & $\mathrm{F}$ & 0 & 0 & 2 & 2 \\
\hline & & $\%$ & $0 \%$ & $0 \%$ & $6 \%$ & $6 \%$ \\
\hline & \multirow{2}{*}{ Total } & $\mathrm{F}$ & 16 & 14 & 6 & 36 \\
\hline & & $\%$ & $44 \%$ & $39 \%$ & $17 \%$ & $100 \%$ \\
\hline
\end{tabular}

Nota. $\mathrm{F}=$ Frecuencia, resultados del procesamiento mediante el programa SPSS. Elaboración propia.

Tabla 3

Distribución de frecuencias y porcentajes del indicador Transparencia con la variable Ejecución Presupuestaria de la Municipalidad Distrital de Lurigancho en el año fiscal 2016

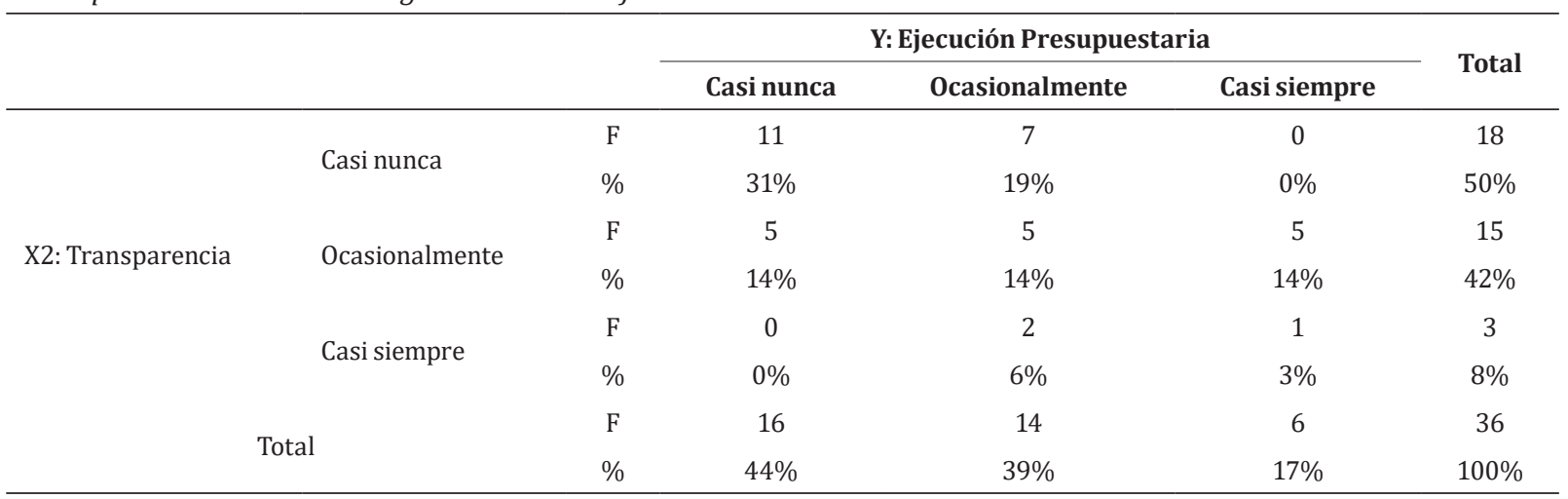

Nota. F= Frecuencia, resultados del procesamiento mediante el programa SPSS. Elaboración propia. 
ejecuta el presupuesto; un 14\% respondió que ocasionalmente se aplica la ejecución presupuestaria. Se finaliza mencionando que, solo el $3 \%$ afirma que la ejecución presupuestaria casi siempre se aplica.

Se evidencia en la Tabla 4, que el 50\% contestó que la igualdad casi nunca es aplicada, seguido de un $36 \%$ contestaron que ocasionalmente se aplica la igualdad, el $11 \%$ que dicha igualdad que casi siempre se aplica, ahora bien, un 3\% dijo que se aplica siempre la igualdad, de igual forma el $44 \%$ afirma que la ejecución presupuestaria casi nunca es utilizada, un 33\% indica que la ejecución presupuestaria es ocasionalmente empleada; de igual forma, solo un $11 \%$ refiere que la ejecución presupuestaria casi siempre se aplica. Finalmente, un 3\% dijo que la ejecución presupuestaria siempre se utiliza.

En la Tabla 5, se puede evidenciar que existe una alta correlación entre el "Presupuesto Participativo y la ejecución presupuestaria de la Municipalidad Distrital de Lurigancho en el ejercicio fiscal 2016" ( $r=.952)$; asimismo resalta que la Participación posee una alta correlación

Tabla 4

Distribución de frecuencias y porcentajes del indicador Igualdad con la variable Ejecución Presupuestaria de la Municipalidad Distrital de Lurigancho en el año fiscal 2016

\begin{tabular}{|c|c|c|c|c|c|c|}
\hline & & & \multicolumn{3}{|c|}{ Y: Ejecución Presupuestaria } & \multirow{2}{*}{ Tota } \\
\hline & & & Casi nunca & Ocasionalmente & Casi siempre & \\
\hline \multirow{10}{*}{ X3: Igualdad } & \multirow{2}{*}{ Casi nunca } & $\mathrm{F}$ & 16 & 2 & 0 & 18 \\
\hline & & $\%$ & $44 \%$ & $6 \%$ & $0 \%$ & $50 \%$ \\
\hline & \multirow{2}{*}{ Ocasionalmente } & $\mathrm{F}$ & 0 & 12 & 1 & 13 \\
\hline & & $\%$ & $0 \%$ & $33 \%$ & $3 \%$ & $36 \%$ \\
\hline & \multirow{2}{*}{ Casi siempre } & $\mathrm{F}$ & 0 & 0 & 4 & 4 \\
\hline & & $\%$ & $0 \%$ & $0 \%$ & $11 \%$ & $11 \%$ \\
\hline & \multirow{2}{*}{ Siempre } & $\mathrm{F}$ & 0 & 0 & 1 & 1 \\
\hline & & $\%$ & $0 \%$ & $0 \%$ & $3 \%$ & $3 \%$ \\
\hline & \multirow{2}{*}{ Total } & $\mathrm{F}$ & 16 & 14 & 6 & 36 \\
\hline & & $\%$ & $44 \%$ & $39 \%$ & $17 \%$ & $100 \%$ \\
\hline
\end{tabular}

Nota. F= Frecuencia, resultados del procesamiento mediante el programa SPSS. Elaboración propia.

Tabla 5

Correlación entre las variables del Presupuesto Participativo con la ejecución presupuestaria de la Municipalidad Distrital de Lurigancho en el año fiscal 2016

\begin{tabular}{|c|c|c|c|}
\hline Variable & & & $\mathbf{Y}$ \\
\hline \multirow{12}{*}{ Rho de Spearman } & \multirow{3}{*}{$\mathrm{X}$} & Coeficiente de correlación & $.952^{* *}$ \\
\hline & & Sig. (bilateral) & .000 \\
\hline & & $\mathrm{N}$ & 36 \\
\hline & \multirow{3}{*}{$\mathrm{X} 1$} & Coeficiente de correlación & $.925^{* *}$ \\
\hline & & Sig. (bilateral) & .000 \\
\hline & & $\mathrm{N}$ & 36 \\
\hline & \multirow{3}{*}{$\mathrm{X} 2$} & Coeficiente de correlación & $.554^{* *}$ \\
\hline & & Sig. (bilateral) & .000 \\
\hline & & $\mathrm{N}$ & 36 \\
\hline & \multirow{3}{*}{ X3 } & Coeficiente de correlación & $.979^{* *}$ \\
\hline & & Sig. (bilateral) & .000 \\
\hline & & $\mathrm{N}$ & 36 \\
\hline
\end{tabular}

**. La correlación es significativa en el nivel 0,01 (bilateral).

Nota. Resultados del procesamiento mediante el programa SPSS. Elaboración propia. 
con la Ejecución Presupuestaria ( $\mathrm{r}=.925)$. Por otro lado, se evidencia que entre la Transparencia y la Ejecución Presupuestaria, existe una moderada correlación $(\mathrm{r}=.554)$, a diferencia que la Igualdad y la Ejecución Presupuestaria, existe una alta correlación $(\mathrm{r}=.979)$.

\section{DISCUSIÓN}

En el presente artículo se encontró que entre la variable Presupuesto Participativo y la variable Ejecución Presupuestaria existe una alta correlación.

Se corrobora la relación alta y estadísticamente significativa entre Participación y Ejecución Presupuestaria $(r=.925)$. Esto es consecuente con lo hallado por Gambini (2011), sobre la importancia de la inclusión de las personas en el manejo del presupuesto y consigo la satisfacción ante las necesidades básicas, tal como lo señala Sánchez (2000), que dicha intervención en las actividades mejora la calidad de vida de la sociedad en manera conjunta.

Por otro lado, la correlación entre la variable Ejecución Presupuestaria y Transparencia fue estadísticamente significativa y de grado moderado $(\mathrm{r}=.554)$. Tal como señala Agurcia (2011) sobre la obligación de las instituciones en informar por ley sobre los proyectos a tratar, de tal manera que el vínculo entre el crecimiento y la inversión pública se ejecuten a la par y de manera clara (Gambini, 2011; Tabuca, 2016), sin influencia externa (Rojas, 2015).

La relación entre Igualdad y Ejecución Presupuestaria fue estadísticamente significativa $y$ de grado alto $(r=.979)$. A partir de ello, se entiende que, ante la diversidad y sistemas políticos, se debe poseer las mismas oportunidades para acceder al bienestar, dentro de un Estado que tiene presente la democracia (Montoya, 2007; Fun. Luis Vives, 2010)

Finalmente, la poca participación de los agentes en el proceso del presupuesto participativo, la limitada comunicación sobre los proyectos priorizados en dicho proceso y la capacitación de los agentes que no se da en forma uniforme por parte del equipo técnico; dificultan el desarrollo de la Ejecución Presupuestaria del Presupuesto Participativo en la Municipalidad Distrital de Lurigancho.

\section{CONCLUSIONES}

Se estableció que, entre el Presupuesto Participativo y la Ejecución Presupuestaria del Municipio de Lurigancho, en el ejercicio 2016, existe una alta correlación $[0,952]$. Dicho en otras palabras, el presupuesto participativo se relaciona con las modificaciones presupuestarias de igual manera con los gastos e ingresos públicos.

Se determinó que, la Participación y la Ejecución Presupuestaria del Municipio de Lurigancho, en el ejercicio 2016, tienen una alta correlación $[0,925]$. Es decir que la Participación se relaciona con las modificaciones presupuestarias, del mismo modo con los ingresos públicos y gastos públicos.

Se puntualizó que, la Transparencia y la Ejecución Presupuestaria del Municipio de Lurigancho, en el ejercicio 2016, tienen una moderada correlación [0,554]. Es decir que la Transparencia tiene una moderada correlación con las modificaciones presupuestarias, del mismo modo con los ingresos públicos y gastos públicos.

Se constató que, la Igualdad y la Ejecución Presupuestaria del Municipio de Lurigancho, en el ejercicio 2016, tienen de una alta correlación $[0,979]$. De esta manera de constata la alta correlación que la Igualdad tiene una con las modificaciones presupuestarias, de igual manera con los ingresos públicos y gastos públicos.

\section{REFERENCIAS BIBLIOGRÁFICAS}

Agurcia, G. (2011). Importancia del Derecho de Acceso a la Información Pública. La Revista de Derecho, 32(1). Obtenido de https://bit.ly/2MTihjZ

Blanco, F. I. (8 de octubre de 2002). Presupuestos Participativos y Democracia Local: Una Comparación Entre las Experiencias Brasileñas y Españolas. VII Congreso Internacional del CLAD, sobre la reforma del Estado y de la Administración Pública. Obtenido de https://bit.ly/20K1TiJ

Decreto Legislativo. $N^{\circ} 1440$. (16 de Setiembre de 2018). Decreto Legislativo $\mathrm{N}^{\circ}$ 1440. Decreto Legislativo del Sistema Nacional de Presupuesto Público, Normas Legales. Lima, Lima, Perú: EDITORA PERU. Obtenido de http://bit. ly/20w $2 \mathrm{hBb}$ 
Fun. Luis Vives. (2010). Claves sobre la Igualdad y la no Discriminación. Madrid. Obtenido de https:// bit.ly/2wJ7QV6

Gambini, G. (2011). La participación ciudadana en la Gestión del Presupuesto Participativo y su Influencia en el nivel de satisfacción de la población del Distrito de Gregorio Albarracin Lanchipa (2007-2009). (Tesis de Maestría), Universidad Nacional Jorge Basadre Grohman, Tacna, Perú. Obtenido de https://bit.ly/2pplLMT

Ley $\mathrm{N}^{\circ}$ 28056. (8 de agosto de 2003). Ley Marco del Presupuesto Participativo. Diario Oficial El Peruano, págs. 249441-249442. Obtenido de http://bit.ly/2yWfKhr

Linale, V. (2006). Uruguay Educa - Un portal en movimiento. Obtenido de Participación. Conceptos y formas: http://bit.ly/2oXfKa8

Makón, M. (1999). Sistemas Integrados de Administración Financiera en América Latina. Dirección de Programación del Sector Público. Argentina.

Montoya, A. (2007). La Igualdad como valor, como principio y como derecho fundamental. ARANZADI. Obtenido de https://bit.ly/2NPU7mV
Páez-Álvarez A. (2006). La participación ciudadana y su relación con el acceso a la información pública. Ra Ximhai (2) 3, 611-640. Obtenido de https://www.redalyc.org/pdf/461/46120304. pdf

Rojas, C. R. (2015). Factores que limitan la participación ciudadana en el Presupuesto Participativo. Caso: Distrito de Mariano Melgar. (Tesis de Maestría), Pontificia Universidad Católica del Perú (PUCP), Lima, Perú. Obtenido de https:// bit.ly/20sOBe1

Sánchez, E. (2000). Continuidad de la participación comunitaria. La definición de Participación. Carcas. Obtenido de https://bit.ly/2cxNkKQ

Tabuca, A. (30 de diciembre de 2016). Las funciones del presupuesto público en la economía. Revista Universitaria Ruta, 18(2). Obtenido de https:// bit.ly/2lgW1QN

UN-HABITAT. (2004). ¿Qué es y cómo se hace el Presupuesto Participativo? 72 respuestas a Preguntas Frecuentes sobre Presupuestos Participativo Municipales. Quito: AH Editorial. Obtenido de http://bit.ly/2yvmwdl 